Full length article

\title{
Multiuser detection for the uplink of clustered 5G systems with universal frequency reuse
}

\author{
F. Casal Ribeiro ${ }^{a, b, *}$, R. Dinis ${ }^{a, c}, F \cdot$ Cercas $^{a, b}, A$. Silva ${ }^{a, d}$ \\ ${ }^{a}$ IT - Instituto de Telecomunicações, Portugal \\ ${ }^{\mathrm{b}}$ ISCTE - Instituto Universitário de Lisboa, Portugal \\ ${ }^{c}$ FCT - Universidade Nova de Lisboa, Portugal \\ ${ }^{\mathrm{d}}$ DETI - Universidade de Aveiro, Portugal
}

\section{A R T I C L E I N F O}

\section{Article history:}

Received 6 January 2016

Received in revised form

4 November 2016

Accepted 20 January 2017

Available online 24 January 2017

\section{Keywords:}

Universal frequency reuse

BS cooperation

C-RAN

SC-FDE

IB-DFE

\begin{abstract}
A B S T R A C T
With 5G (Fifth generation) cellular communications, systems have to be able to cope with a massive increase of mobile devices and services and simultaneously improve the system's spectral efficiency, as well as dealing with high interference levels. Base Station (BS) cooperation architectures jointly with block transmission techniques, such as OFDM (Orthogonal Frequency Division Multiplexing) for the downlink and SC-FDE (Single-Carrier with Frequency-Domain Equalization) for the uplink, are proven to be suitable for broadband wireless transmission systems. In BS cooperation systems MTs (Mobile Terminals) in adjacent cells share the same physical channel allowing the reducing of the frequency reuse and improving the spectral efficiency of cellular systems. In this paper we present a set of multiuser detection techniques for the uplink transmission in clustered architectures based on the C-RAN (Centralized-Radio Access Network) concept. We consider BS cooperation systems employing a universal frequency reuse approach. Our performance results demonstrate that by employing clustered techniques for the detection procedure it is possible to reduce substantially the signal processing complexity and the side information that must be transmitted by the backhaul structure.
\end{abstract}

(C) 2017 Elsevier B.V. All rights reserved.

\section{Introduction}

The ever increase of wireless connected devices and services will pressure the capacity and quality of near future cellular infrastructures. When compared to the current 4G (Fourth Generation) LTE (Long-Term Evolution) systems, it is expected that 5G (Fifth Generation) cellular communications can deal with the massive explosion of mobile devices, the need for higher data transmissions, spectral efficiency and high-speed mobility users [1]. This will be achieved mainly by combining massive MIMO (Multi-Input, Multi-Output) techniques, small cells and employing reduced frequency reuse factors (ideally aiming at a universal frequency reuse) [2]. Moreover, one can consider CRAN (Centralized-Radio Access Network) architectures as one of the optimal approaches to enhance the potential of BS (Base Station) cooperation schemes [3-5], which are critical to cope with high interference levels associated to reduced frequency reuse

\footnotetext{
* Corresponding author at: ISCTE - Instituto Universitário de Lisboa, Portugal.

E-mail address: casalribeiro.filipe@gmail.com (F.C. Ribeiro).
}

factors [6]. Contrarily to BS cooperation schemes, conventional architectures assign different frequency bands for different BSs, leading to a substantial degradation on the overall system's spectral efficiency. Due to the physical scarcity of the frequency spectrum it is highly desirable to have universal frequency reuse. By implementing BS cooperations schemes, MTs (Mobile Terminals) in adjacent cells can employ the same physical channel, allowing substantial capacity gains when compared with conventional systems. Thus, a set of BSs might need to exchange information so as to define appropriate transmitted signals (in the downlink transmission) [7] and the detection or/and users separation being performed by a CPU (Central Processing Unit) connected to several BSs (in the uplink transmission) $[8,9]$. Furthermore, in the downlink case it is preferable to employ appropriate preprocessing techniques so as allow an efficient separation of the signals at the receiver side [10]. For the uplink transmission, one can perform the separation of different users at the receiver side, since different BS can easily cooperate at the CRAN level [6,8].

Block transmission techniques, jointly with frequency-domain processing methods [11], are particularly adequate for broad- 
band wireless systems. It is well known that SC-FDE (SingleCarrier with Frequency-Domain Equalization) modulations are particularly suitable for the uplink transmission since the reduced envelope fluctuations of the transmitted signals, namely when compared with OFDM (Orthogonal Frequency Division Multiplexing) signals based on similar constellations, allow an efficient power amplification [12-14]. At the receiver side, the separation of the signals associated with different MTs and/or interference mitigation is performed using iterative frequency-domain receivers based on the IB-DFE (Iterative Block Decision Feedback Equalization) design $[15,16]$, allowing performance results close to the MFB (Matched Filter Bound) measure [17], even in strongly interference environments. Moreover, IB-DFE receivers can be regarded as frequency-domain low complexity turbo equalizers $[18,19]$, in which it does not require the channel decoder output at the feedback loop. Nevertheless, the receiver complexity and the global quantity of signals that are required to be exchanged by the backhaul network for the user separation increases with the number of BSs that are cooperating. The adequate dealing of this problem is to consider clustered schemes to effectively reduce both the detection complexity and the backhaul signaling requirements. In [20] a study on the uplink of BS cooperation schemes is presented. However, this paper approaches the scalability issues by considering clustered environments.

In this paper we consider the uplink transmission in BS cooperation cellular architectures, with universal frequency reuse and SC-FDE transmission schemes. The concept of BS cooperation is related to C-RAN type environments, in which the implementation of clustered techniques are intended to simplify the detection procedure and to reduce the side information shared by the BSs.

This paper is organized as follows: in Section 2 we describe the clustered cellular architecture considered in this paper and Section 3 is concerned with the receiver design. Section 4 describes the different detection techniques and a set of performance results is presented in Section 5. Section 6 concludes the paper.

Throughout the paper we will adopt the following notations: bold letters denote vectors and matrices; $\mathbf{x}^{*}, \mathbf{x}^{T}$ and $\mathbf{x}^{H}$ denote complex conjugate, transpose and Hermitian (complex conjugate transpose) of $\mathbf{x}$, respectively. $\mathbf{I}_{N}$ denotes a $N \times N$ identity matrix and $\mathbf{e}_{p}$ is an appropriate column vector with 0 in all positions except the $p$ th position that is 1 . The expectation of $x$ is denoted by $\mathbb{E}[x]$.

\section{System characterization}

Fig. 1 represents the adopted cellular scenario considered in this paper. The system is characterized by two clusters employing BS cooperation-based wireless architectures. Both clusters can communicate through a backhaul network in a C-RAN structure and we consider inter-cluster interference that is accounted at each BS. BS cooperation systems are characterized by the use of a CPU that performs interference mitigation and/or user detection functions, allowing the different MTs to transmit information in the same frequency. Each cluster can have $P$ MTs sharing the same physical channel and communicating to $R$ BSs. Each MT employs a SC-FDE modulation scheme with an appropriate $\mathrm{CP}$ being appended to each data block. Assuming that initially we have free inter-cluster interference, if the $\mathrm{CP}$ (Cyclic Prefix) has the correct length, at a given $\mathrm{BS} r$, the received signal is given by

$Y_{k}^{(r)}=\sum_{p=1}^{P} S_{k, p} H_{k, p}^{e p(r)}+N_{k}^{(r)}$,

where $\left\{Y_{k}^{(r)} ; k=0,1, \ldots, N-1\right\}$ is the DFT (Discrete Fourier Transform) of the useful time-domain block (i.e., after removing the samples associated to the cyclic prefix) $\left\{y_{n}^{(r)} ; n=0,1, \ldots, N-\right.$
1\} $(r=1,2, \ldots, R)$. Moreover, $S_{k, p}=$ DFT $\left\{s_{n, p} ; n=0,1, \ldots, N-\right.$ $1\}$, associated to the $p$ th MT $(p=1,2, \ldots, P)$ and the constellation symbol $\left\{s_{n, p} ; n=0,1, \ldots, N-1\right\}$ is selected following a given mapping rule such as QPSK (Quadrature Phase Shift Keying) with Gray mapping. $N_{k}^{(r)}$ indicates the channel noise at the $r$ th antenna and the $k$ th frequency and $H_{k, p}^{e q(r)}=\xi_{p, r} H_{k, p}^{(r)}$, where $H_{k, p}^{(r)}$ denotes the channel frequency response between the $p$ th MT and the $r$ th BS, for the $k$ th frequency. The coefficient $\xi_{p, r}$ corresponds to a factor that accounts for both effects of power control and propagation losses. Therefore, the average received power at the $r$ th BS associated to the $p$ th MT is $\left|\xi_{p, r}\right|^{2}$, with a normalized channel frequency response as $\mathbb{E}\left[\left|H_{k, p}^{(r)}\right|^{2}\right]=1$.

When a single cluster is considered, all transmission contributions can be taken into account, rewriting (1) as

$\mathbf{Y}_{k}=\mathbf{H}_{k}^{T} \mathbf{S}_{k}+\mathbf{N}_{k}$,

with $\mathbf{Y}_{k}=\left[Y_{k}^{(1)}, \ldots, Y_{k}^{(R)}\right]^{T}, \mathbf{S}_{k}=\left[S_{k, 1}, \ldots, S_{k, P}\right]^{T}, \mathbf{N}_{k}=$ $\left[N_{k}^{(1)}, \ldots, N_{k}^{(R)}\right]^{T}$ and

$\mathbf{H}_{k}^{T}=\left[\begin{array}{ccc}H_{k, 1}^{e q^{(1)}} & \cdots & H_{k, P}^{e q^{(1)}} \\ \vdots & \ddots & \vdots \\ H_{k, 1}^{e q^{(R)}} & \cdots & H_{k, P}^{e q^{(R)}}\end{array}\right]$.

\section{Receiver structure}

The multiuser detection process is performed by a structure based on the IB-DFE concept [16] and proposed in [20], where this receiver can be regarded as a specific implementation scheme for BS cooperation architectures. Each iteration provides a more recent estimation for the transmitted data symbols for a given MT being detected, which are used to cancel the residual interference inherent to the detection process. Moreover, users are detected in a successive methodology, which can be seen as an iterative SIC (Successive Interference Cancellation) scheme. When a certain $p$ th MT is detected at the $i$ th iteration, the frequency-domain detector output is expressed as

$\tilde{S}_{k, p}^{(i)}=\mathbf{F}_{k, p}^{(i)^{T}} \mathbf{Y}_{k}-\mathbf{B}_{k, p}^{(i)} \overline{\mathbf{S}}_{k, p}^{(i-1)}$.

The most recent estimation symbols $\left\{\hat{s}_{n, p}^{(i)} ; n=0,1, \ldots, N-1\right\}$, $(p=1,2, \ldots, P)$ correspond to hard decisions of the time-domain detector output $\left\{\tilde{s}_{n, p}^{(i)} ; n=0,1, \ldots, N-1\right\},(p=1,2, \ldots, P)$. $\mathbf{F}_{k, p}^{(i)^{T}}=\left[F_{k, p}^{(i, 1)}, \ldots, F_{k, p}^{(i, R)}\right]^{T}$ and $\mathbf{B}_{k, p}^{(i) T^{T}}=\left[B_{k, p}^{(i, 1)}, \ldots, B_{k, p}^{(i, P)}\right]^{T}$ denote the feedforward and feedback coefficients, respectively. $\overline{\mathbf{S}}_{k, p}^{(i-1)}=\left[\bar{S}_{k, 1}^{(i)}, \ldots, \bar{S}_{k, p-1}^{(i)}, \bar{S}_{k, p}^{(i-1)}, \ldots, \bar{S}_{k, P}^{(i-1)}\right]^{T}$, and its elements are associated to the current iteration for MTs already estimated and the previous iteration for the MT currently being detected, as well as the MTs that were not yet detected in the current iteration.

It can be shown (as in [20]) that the optimum coefficients $\mathbf{F}_{k, p}$ and $\mathbf{B}_{k, p}$ are given by

$\mathbf{F}=\kappa \boldsymbol{\Lambda} \mathbf{H}^{H} \mathbf{e}_{p}$

and

$\mathbf{B}=\mathbf{H F}-\mathbf{e}_{p}$,

with

$\boldsymbol{\Lambda}=\left(\mathbf{H}^{H}\left(\mathbf{I}_{P}-\mathbf{P}^{2}\right) \mathbf{H}+f(S N R) \mathbf{I}_{R}\right)^{-1}$, 


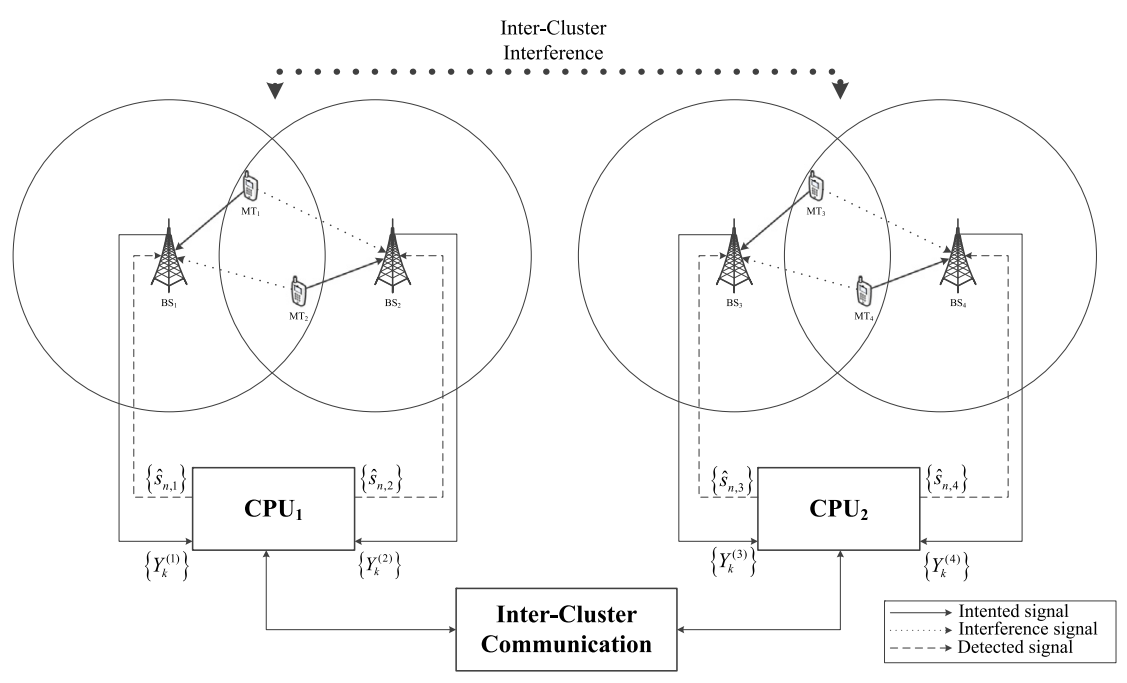

Fig. 1. Adopted cellular scenario.

and $\kappa$ selected to ensure that

$\frac{1}{N} \sum_{k=0}^{N-1} \sum_{r=1}^{R} F_{k, p}^{(r)} H_{k, p}^{e q(r)}=1$.

$f(S N R)$ is a function of the SNR (Signal-to-Noise Ratio) measure, in which

$S N R=\frac{\sigma_{s}^{2}}{\sigma_{n}^{2}}$,

where $\sigma_{s}^{2}$ and $\sigma_{n}^{2}$ are the variance of the signal and noise components, respectively. Furthermore, according to [20], the optimum value for $f$ (SNR) corresponds to 1/SNR.

\section{Multiuser clustered detection techniques}

In this section it is presented a set of multiuser detection techniques for the clustered architecture represented in Fig. 1. The main idea is to perform the MTs detection as to reduce substantially the signal processing complexity and the required side information without sacrificing the performance. Additionally, the detection procedure depends on the interference presented in the system, as if it becomes an impediment for a proper detection, both clusters can iteratively (as with inter-cluster communication) share information in order to cancel the interference. We present three techniques to perform the multiuser detection, differing on the type of consideration we have in terms of interference from one cluster to another. For the multiuser clustered techniques, (1) can be expressed by

$Y_{k}^{(r)}=\sum_{p \in C}^{P} S_{k, p} H_{k, p}^{e p(r)}+\sum_{p \notin C}^{P} S_{k, p} H_{k, p}^{e p(r)}+N_{k}^{(r)}$,

with $C$ denoting the set of BSs in the cluster (e.g., for the cluster with BSs 1 and 2 we have $C=\{1,2\})$. Moreover, we consider two scenarios, one with a fixed separation between clusters and one with a linear set of cells, which corresponds to a more realistic scenario.

\subsection{Fixed separation between clusters}

Fig. 2 illustrate a 2 cells scenario with limited inter-cell links, where $\beta$ indicates the interference from a given MT to the remaining BSs in the other cluster. We assume that the links belonging to a given cluster have a transmission reference value (i.e., the main link) of 1 (or $0 \mathrm{~dB}$ ). Moreover, $\beta$ indicates the average power below the main link. For simplicity purposes, it is only shown the $\beta$ effect from the 1st MT. The case for the remaining MTs can easily be extended.

\subsubsection{SCD (Single Cluster Detection)}

SCD (Single Cluster Detection) corresponds to a localized detection with no sharing of information between clusters. It provides the lowermost system requirements, in which for detection purposes, despite the $\beta$ interference, the system is composed of $P=2$ MTs and $R=2$ BSs. The power associated with the different links $\xi_{p, r}$ is given by

$\boldsymbol{\Xi}_{C}=\left[\begin{array}{ll}\xi_{1,1} & \xi_{1,2} \\ \xi_{2,1} & \xi_{2,2}\end{array}\right]=\left[\begin{array}{ll}1 & 1 \\ 1 & 1\end{array}\right]$.

Nevertheless it is also taken into account the interference originated from the remaining MTs for detection purposes. For localized detections in clusters scenarios, $\mathbf{H}_{k}$, firstly defined in (3), is written according to the cluster that is being considered. So, for the case in (11) we only consider $\mathbf{H}_{C}$ from the complete $\mathbf{H}_{k}$ matrix form. Therefore, the $\mathbf{F}$ and $\mathbf{B}$ coefficients are given by

$\mathbf{F}_{C}=\kappa \boldsymbol{\Lambda}_{C} \mathbf{H}_{C}^{H} \mathbf{e}_{2}$

and

$\mathbf{B}_{C}=\mathbf{H}_{C} \mathbf{F}_{C}-\mathbf{e}_{2}$,

with

$\mathbf{\Lambda}_{C}=\left(\mathbf{H}_{C}^{H}\left(\mathbf{I}_{2}-\mathbf{P}_{2}^{2}\right) \mathbf{H}_{C}+f(S N R) \mathbf{I}_{2}\right)^{-1}$.

\subsubsection{FD (Full Detection)}

The FD (Full Detection) procedure is employed with total sharing of information. The system can be seen as a single cluster with $P=R=4$ and it presents macro-diversity effects inherent to BS cooperation architectures and the power associated with the different links $\xi_{p, r}$ can be written as

$\boldsymbol{\Xi}=\left[\begin{array}{llll}\xi_{1,1} & \xi_{1,2} & \xi_{1,3} & \xi_{1,4} \\ \xi_{2,1} & \xi_{2,2} & \xi_{2,3} & \xi_{2,4} \\ \xi_{3,1} & \xi_{3,2} & \xi_{3,3} & \xi_{3,4} \\ \xi_{4,1} & \xi_{4,2} & \xi_{4,3} & \xi_{4,4}\end{array}\right]=\left[\begin{array}{llll}1 & 1 & \beta & \beta \\ 1 & 1 & \beta & \beta \\ \beta & \beta & 1 & 1 \\ \beta & \beta & 1 & 1\end{array}\right]$ 


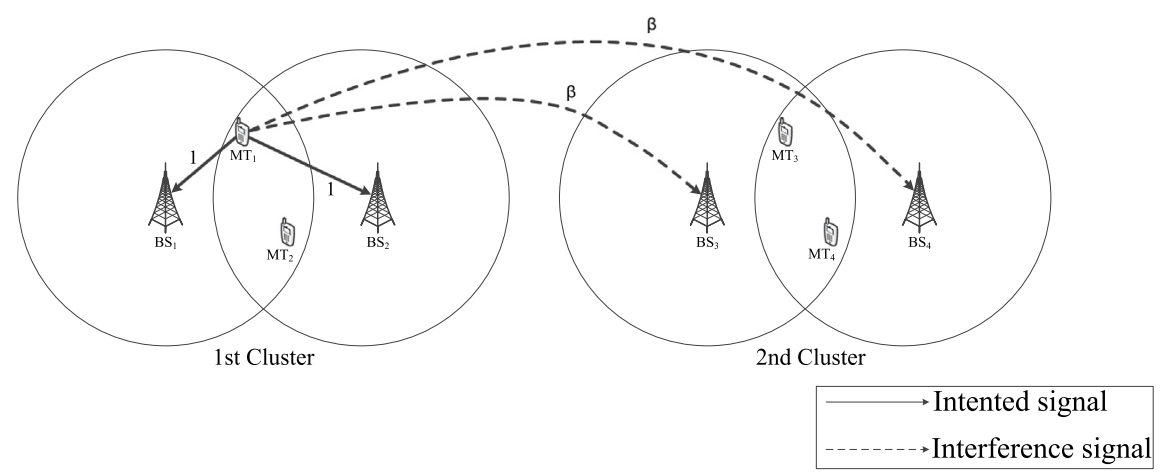

Fig. 2. 1st MT transmission contributions.

\subsection{3. $H D$ (Hybrid Detection)}

The HD (Hybrid Detection) method corresponds to an intermediate scheme for the detection procedure. By enabling the HD approach we can have higher interference values given by $\beta$. This increases the detection requirements in comparison with SCD and decreases regarding FD. In this case, the power associated with the different links $\xi_{p, r}$ is given by

$\boldsymbol{\Xi}=\left[\begin{array}{llll}\xi_{1,1} & \xi_{1,2} & \xi_{1,3} & \xi_{1,4} \\ \xi_{2,1} & \xi_{2,2} & \xi_{2,3} & \xi_{2,4}\end{array}\right]=\left[\begin{array}{llll}1 & 1 & \beta & \beta \\ 1 & 1 & \beta & \beta\end{array}\right]$

\subsection{Linear set of cells}

Instead of a limited inter-cell links scenario we can consider a more realistic scenario, shown in Fig. 3. For the sake of simplicity we only show the interference effect (represented by the coefficient $\alpha$ ) originated from the 1st MT. As previously described with the $\beta$ parameter, $\alpha$ indicates the average power below the main link. BSs that are further away will receive lower interference levels when compared with the closest ones. Moreover, there is some overlapping between clusters. In this case, each cluster performs the detection in a localized method and they can iteratively share side information through the backhaul link in order to strategically compensate the higher or lowest amount of information shared. Hence, we have an iterative receiving scheme in each cluster provided by the implementation of BS cooperation systems and an iterative detection strategy with the sharing of information in a C-RAN type environment. Regarding Fig. 3, the power associated with the different links $\xi_{p, r}$ is given by

$\boldsymbol{\Xi}=\left[\begin{array}{cccc}1 & \alpha & \alpha^{2} & \alpha^{3} \\ \alpha & 1 & \alpha & \alpha^{2} \\ \alpha^{2} & \alpha & 1 & \alpha \\ \alpha^{3} & \alpha^{2} & \alpha & 1\end{array}\right]$.

Moreover, for a SCD method, where each cluster detects its MTs, the power associated with the different links is given by the expressions

$\mathbf{\Xi}_{C}=\left[\begin{array}{ll}\xi_{1,1} & \xi_{1,2} \\ \xi_{2,1} & \xi_{2,2}\end{array}\right]=\left[\begin{array}{ll}1 & \alpha \\ \alpha & 1\end{array}\right]$.

Clearly, the complexity of each detection scheme is conditioned by the size of matrices to invert, in which is done for each iteration and subcarrier, and the required overheads for exchanging received signals and data estimates. This is directly related with the iterative algorithm, where the received signals have size $N$ and the data estimation (concerning each inter-cluster iteration), also have size $N$. For the Single Cluster Detection, this must be done for each cluster.
The complexity of the considered receiver is essentially conditioned by the size of the matrices to invert and the required overheads for exchanging received signals and data estimates, which is shown in Table 1.P and $R$ indicate the total of MTs and BSs, respectively, that are being considered in the system. Moreover, $P_{c}$ and $R_{c}$ are the MTs and $\mathrm{BSs}$, respectively, that belong only in a given cluster.

When we consider clustered architectures, the complexity is conditioned by the fact that for each frequency and each iteration it is necessary to invert a matrix with the cluster dimension. Regarding FD approaches it is necessary to invert a matrix with dimensions equal to the number of receiving BSs. For clustered systems, the global iterations required is related with the number of intra-cluster iterations times the number of intercluster iterations, i.e., 3 to 4 intra-cluster iterations times 1 to 3 inter-cluster iterations. Moreover, with the iterative algorithm the received signals have size $N$ and the data estimation (concerning each inter-cluster iteration), also have size $N$. In the FD definition it is only necessary to perform 3 or 4 iterations. When the Single Cluster Detection is enabled, this must be considered to each cluster.

\section{Performance results}

The previous section described different detection techniques in a clustered context, where the interference levels are related with two scenarios, mainly a 2 cells scenario with limited inter-cell links and a more realistic scenario. In this section we present a set of performance results to properly evaluate both cases previously explained.

The data blocks associated with each MT have $N=256$ data symbols, selected from a QPSK constellation under a Gray mapping rule, plus an appropriate cyclic prefix. We considered a multipath channel with 64 symbols-spaced taps and uncorrelated Rayleigh fading on the different multipath components (similar results were observed for other channels with rich multipath propagation). The channels between different transmitting and receiving antennas are assumed uncorrelated. We have perfect synchronization and channel estimation. It is assumed that the useful part of the blocks transmitted by different MTs arrive at each BS simultaneously. In practice, this could be accomplished by employing extended cyclic prefixes, with duration longer than the maximum overall channel impulse response plus the difference between the maximum and minimum propagation delay between MTs and BSs, provided that we have accurate channel estimates.

Let us start by considering the limited inter-cell links scenario (illustrated in Fig. 2). Fig. 4 illustrates the case where we have a BS cooperation scenario employing a FD approach with the interference parameter $\beta$ corresponding to $0 \mathrm{~dB}$. It is clear that there is a significant performance improvement when the received signals associated to different BSs are combined. The performance 


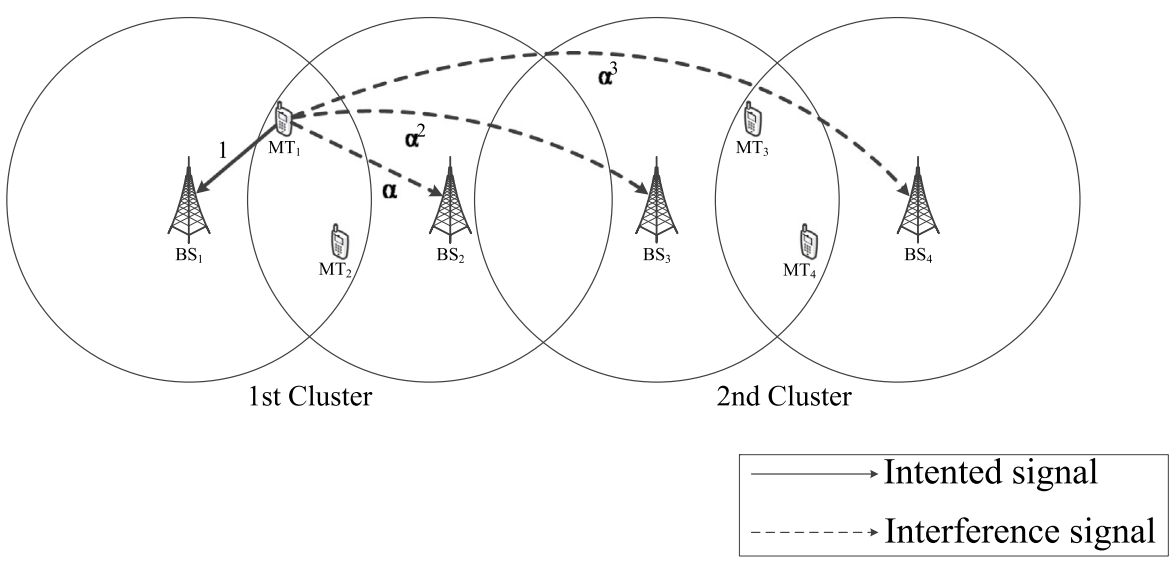

Fig. 3. 1st MT transmission contributions.

Table 1

Complexity and required overheads.

\begin{tabular}{llll}
\hline & Matrix inversion & Required overheads & \\
\cline { 3 - 4 } & Dimensions & Received signals & Data estimates \\
\hline Full Detection & $R \times R$ & $R$ & 0 \\
Single Cluster Detection & $R_{c} \times R_{c}$ & $R_{c}$ & 0 \\
Hybrid Detection & $R \times R$ & $R$ & $R$ \\
\hline
\end{tabular}

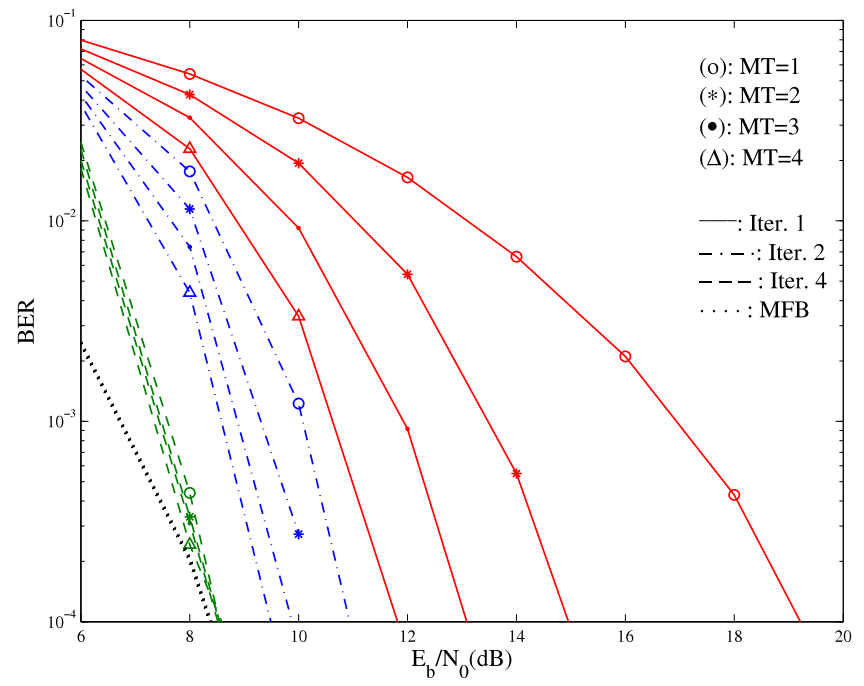

Fig. 4. BS cooperation scenario with $C=1$ clusters, $P=4 \mathrm{MTs}, R=4$ BSs and $\beta=0 \mathrm{~dB}$.

improvement is higher for the 1st iteration, that corresponds to the linear FDE, which is due to the higher residual ISI (Inter-Symbol Interference) at the FDE output. Moreover, the performance of the iterative receiver is already close to the MFB after just 4 iterations and the macro-diversity also reduces the shadowing effects and improves overall coverage.

Fig. 5 considers the BER performance when the receiver is based on that described in [20] framed on a clustered scenario. BSs 1 and 2 are associated with one cluster and BSs 3 and 4 to the other, with interference $\beta=-15 \mathrm{~dB}$. From this figure it is clear that we can have relatively good separation for different MTs. However, the performance degrades for high SNR values (i.e., high values of $E_{b} / N_{0}$ ). This is due to the fact that our receiver assumptions does not consider the inter-cluster interference. For low SNR this is not a problem because the noise is much higher than the residual interference. But for high SNR this leads to a mismatched receiver, since the $\mathbf{F}$ and $\mathbf{B}$ coefficients are designed assuming lower interference levels. To overcome this problem, we

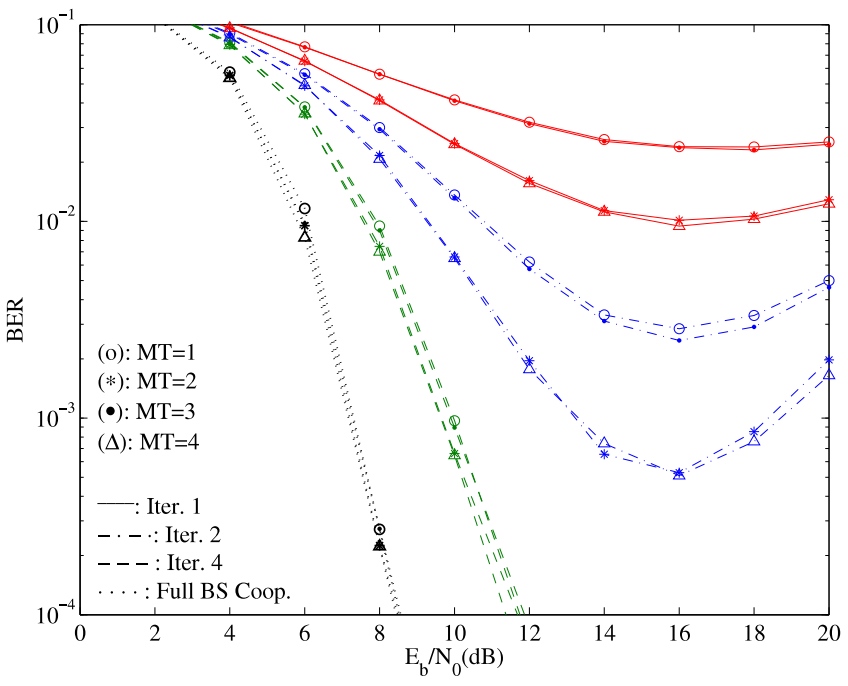

Fig. 5. BER performance for the receiver of [20] in the clustered scenario (one cluster associated to BSs 1 and 2 and the other associated to BSs 3 and 4), when $\beta=-15 \mathrm{~dB}$.

can modify (7) so as to preclude $\sigma_{n}^{2} / \sigma_{s}^{2}=1 /$ SNR taking values too small. This can be done by using $f(S N R)$ as

$f(S N R)= \begin{cases}1 / S N R & \text { if } S N R<S N R_{0} \\ 1 / S N R_{0} & \text { if } S N R \geq S N R_{0}\end{cases}$

instead of the initially $f(S N R)=1 / S N R$ in (7), i.e., by performing a kind of clipping on SNR when we are computing the receiver parameter. We verified $S N R_{0}=13 \mathrm{~dB}$ leads to relatively good results. The corresponding BER performance is depicted in Fig. 6. This value of $S N R_{0}$ was employed in the remaining of the paper. Additionally, the performance results are compared with the case where the FD is employed (i.e., a full BS cooperation detection scheme), since there is not a single definition of MFB for clustered scenarios.

Figs. 7 and 8 show the average BER performance for all MTs, regarding the detection types described in (11) and (16), respectively. In each figure it is shown the performance results 


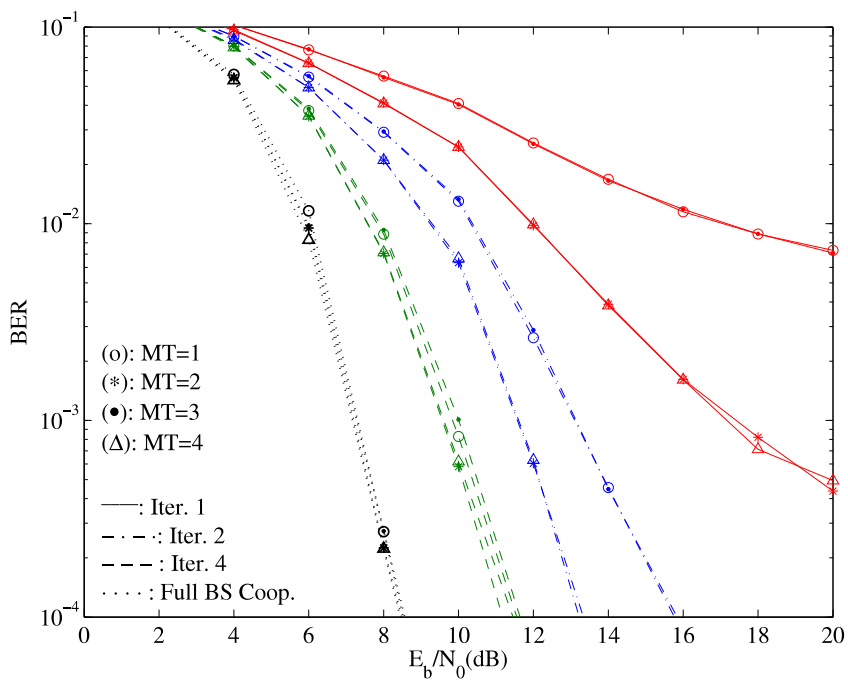

Fig. 6. As in Fig. 5, but setting a limit on the SNR with $S N R_{0}=13 \mathrm{~dB}$ in (19).

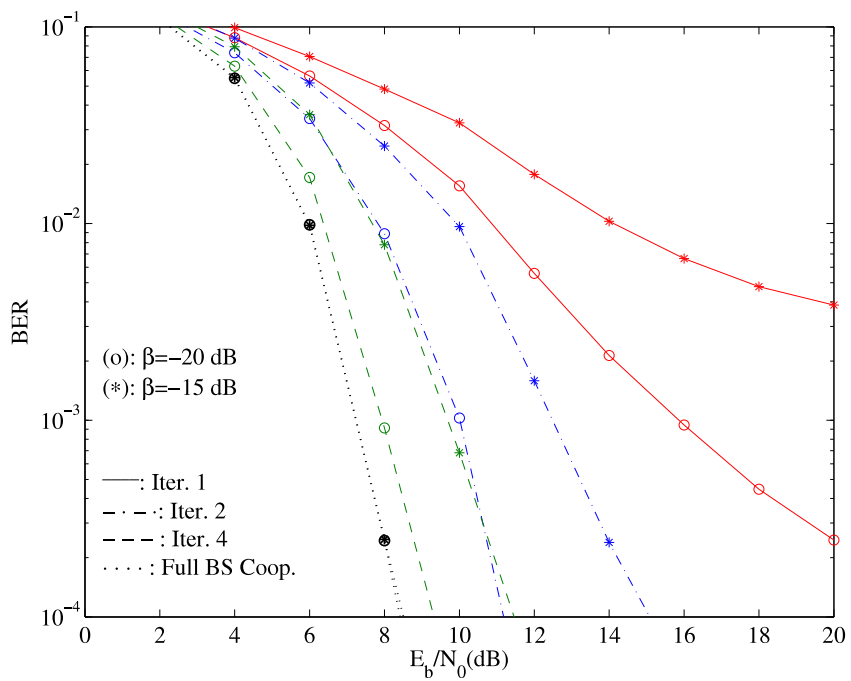

Fig. 7. BS cooperation scenario with $C=2$ clusters, each cluster with $P=2$ MTs and $R=2$ BSs. Detection type based on the SCD approach.

for $\beta$ values of -20 and $-15 \mathrm{~dB}$. Both figures demonstrate that less transmitted power for the interference links induces better performance results. Moreover, it can be seen that there is not a significant difference between the two detection approaches, and in this case it can be considered the detection type regarding the cluster only, which requires less processing.

Let us now analyze the case where 2 clusters communicate to allow interference cancellation and to decrease the overall system's detection requirements. Fig. 9 illustrates the MTs average values for the BER performance results considering the detection based on the single cluster detection method. Clearly, it can be seen that with inter-clusters iterations the BER performance is practically the same of the full BS cooperation case after just 3 iterations. Furthermore, with this approach we can detect the MTs with significant accuracy and decrease the detection requirements, especially when comparing to the results shown in Fig. 4.

Regarding the study of a cellular system based on a more realistic scenario (see Fig. 3), Fig. 10 illustrates the average BER performance from all MTs for each iteration when $\alpha$ corresponds to $-20,-15$ and $-10 \mathrm{~dB}$. As with the scenario previously studied, these results show the impact of higher interference values $(\alpha=-10 \mathrm{~dB})$ on the performance of the considered receiver, when comparing with lower values such as $\alpha=-20 \mathrm{~dB}$.

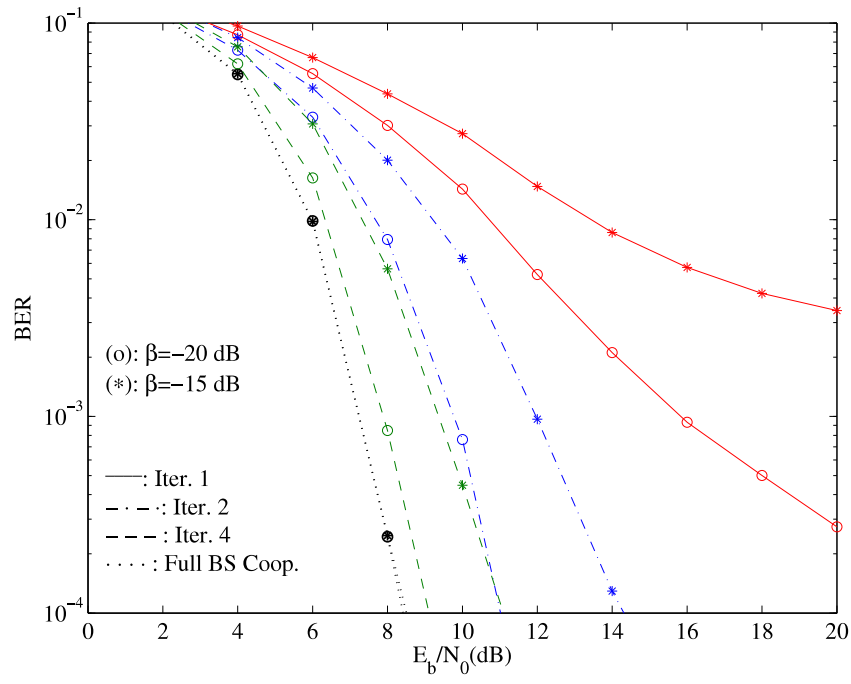

Fig. 8. BS cooperation scenario with $C=2$ clusters, each cluster with $P=2$ MTs and $R=2$ BSs. Detection type based on the HD approach.

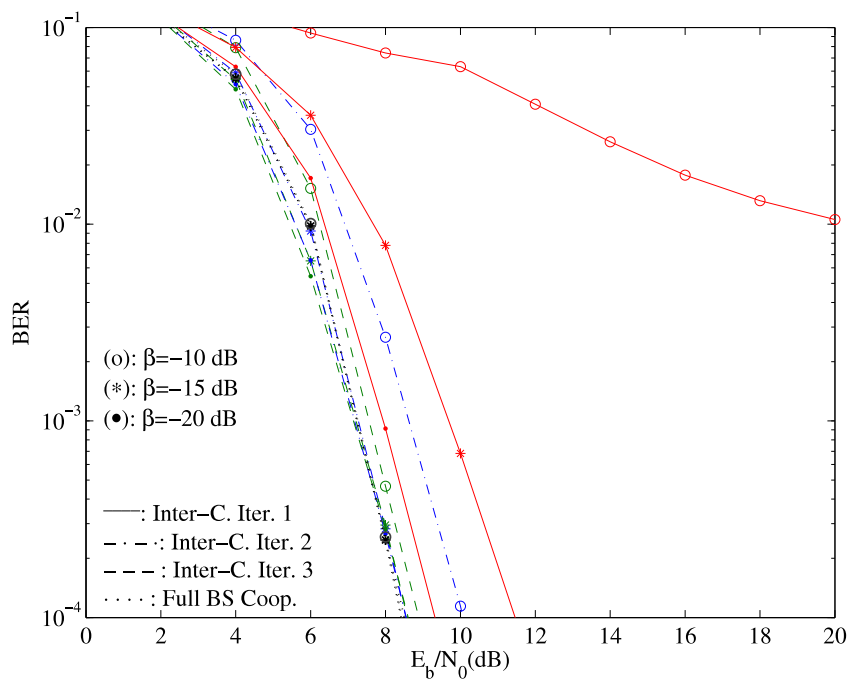

Fig. 9. BS cooperation scenario with $C=2$ clusters, each cluster with $P=2$ MTs and $R=2$ BSs. Detection type based on the SCD approach with inter-cluster communication.

When considering $\alpha=-20 \mathrm{~dB}$, at the fourth iteration, it is already possible to have performance results close to the Full BS cooperation scenario. Considering the scenario presented in Fig. 10, Fig. 11 shows the average iterations that are required for the receiver to perform a successfully detection of a given detected block. Moreover, we also show the PER (Packet Error Rate) associated with the same case. It is possible to see that for high values of $E_{b} / N_{0}$ and considering $\alpha=-20 \mathrm{~dB}$ only one iteration is required, decreasing greatly the process involved to perform the detection. For low values of $E_{b} / N_{0}$ the receiver had to perform 4 iterations, even though it was not sufficient to successfully detect the majority of the blocks, as we can conclude from the PER performance. Nevertheless, it is possible to practically achieve the performance provided by the Full BS cooperation case.

Enabling the sharing of information between clusters in a iterative methodology, Fig. 12 shows the 4th iteration of the average BER performance for all MTs considering the possibility of having inter-cluster iterations. The $\alpha$ values considered are -10 , -5 and $-3 \mathrm{~dB}$. Similarly to the case where we have limited intercell links, by increasing the number of inter-cluster iterations the performance results can be very close to the Full BS cooperation 


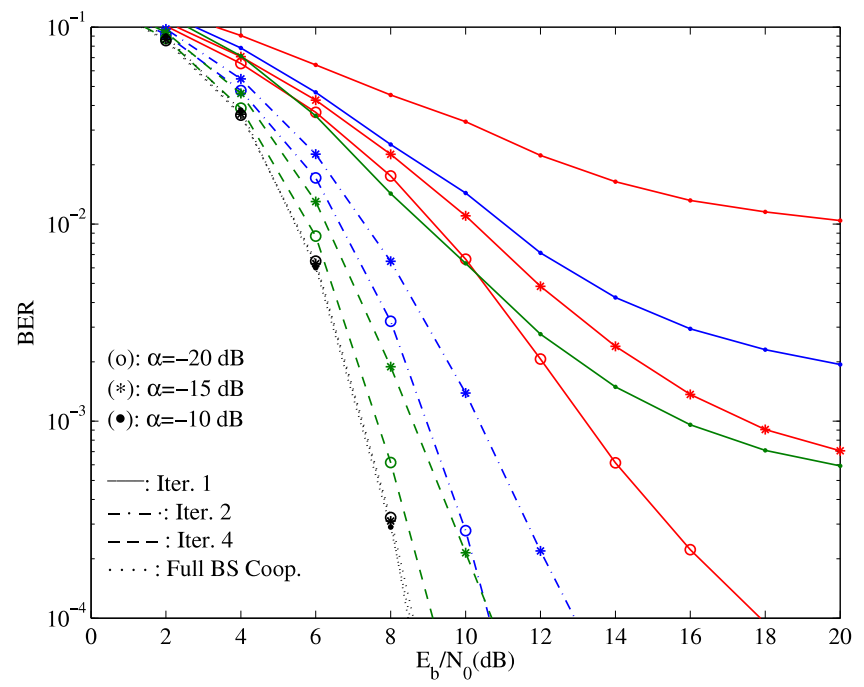

Fig. 10. Average BER performance of all MTs for each iteration, when $\alpha=-20$, -15 and $-10 \mathrm{~dB}$.

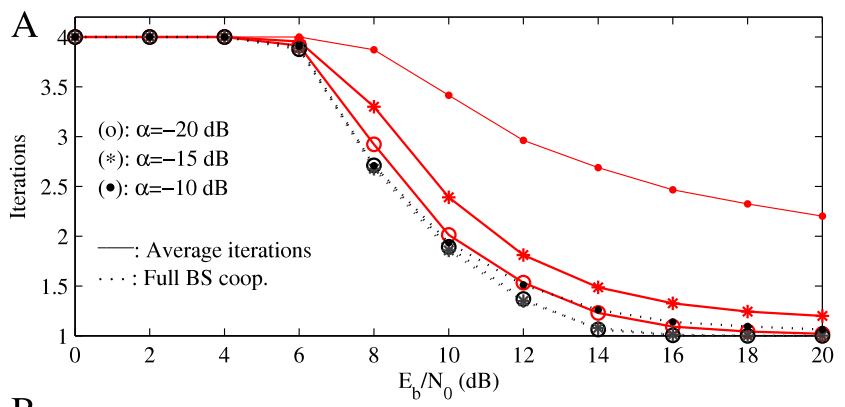

B

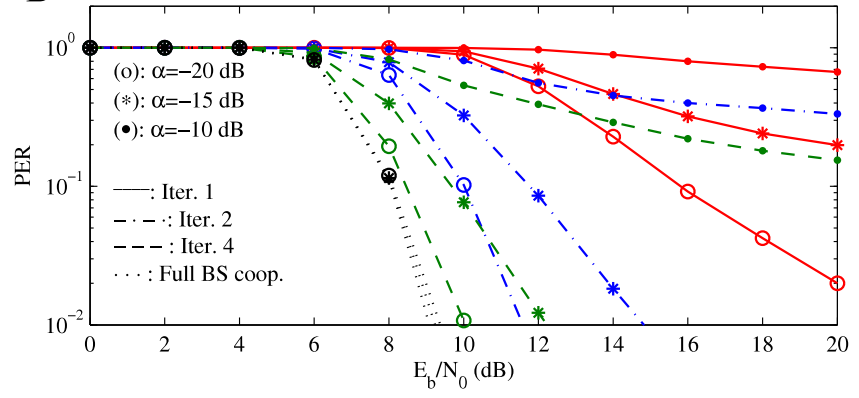

Fig. 11. Average iterations required for successfully detect a given block (A) and average PER (B) performance, in the conditions of Fig. 10.

scenario, even for high values of $\alpha$ such as $-5 \mathrm{~dB}$. Nevertheless, for $\alpha=-3 \mathrm{~dB}$ the performance is largely degraded. So, it is possible to sustain high interference environments by increasing the processing of the iteration method. Taken into account the same scenario presented in Fig. 12 let us consider the average intercluster iterations required to perform a successfully detection in a given block and the PER performance regarding the same case. These results are depicted in Fig. 13, where we considered $\alpha$ values of $-10,-5$ and $-3 \mathrm{~dB}$. As expected, for $\alpha=-10 \mathrm{~dB}$ we only need approximately 1 iteration for a high $E_{b} / N_{0}$. For lower values of $E_{b} / N_{0}$, regardless of the $\alpha$ interference we need 3 inter-cluster iterations, which requires an overload of backhaul information shared between clusters. Moreover, even with 3 iterations the detector was not able to successfully perform the detection, as confirmed by the PER performance.

It should be pointed out that for very large systems the use of the FD technique is too complex, and our clustered techniques can be a very interesting alternative. However, if the interference

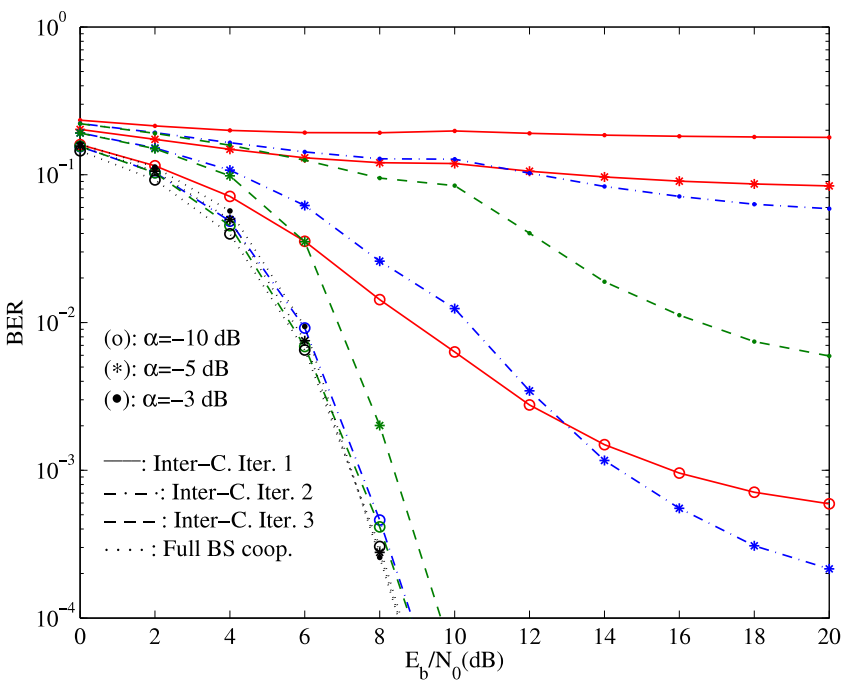

Fig. 12. 4th iteration of average BER performance for all MTs, when $\alpha=-10,-5$ and $-3 \mathrm{~dB}$ and up to 3 inter-cluster iterations.
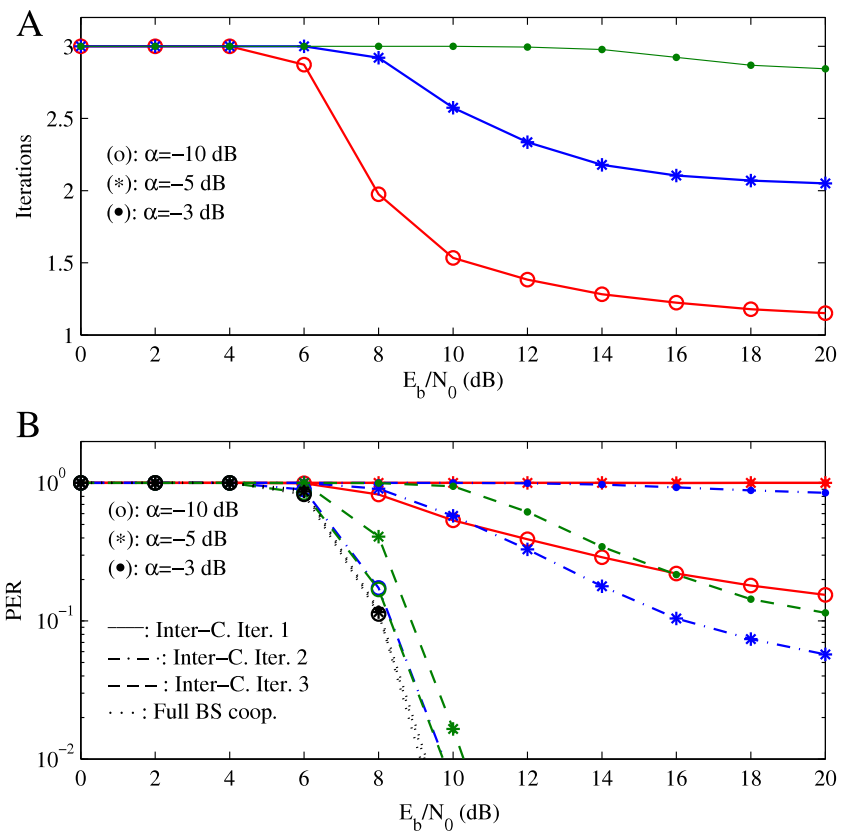

Fig. 13. Average inter-cluster iterations required for successfully detect a given block (A) and average PER performance (B), for each inter-cluster iteration, in the conditions of Fig. 12.

levels are still significative, then it must be necessary to combine our techniques with other techniques, for reducing the number of interfering cells. On the other hand, if the system is operating at very high frequencies (e.g., for mm-wave communications), physical obstacles such as walls can be enough to reduce the number of cells with strong interfering levels, allowing the direct employment of our clustered detection schemes.

\section{Conclusions}

In this paper we considered the uplink transmission in wireless cellular systems in C-RAN type environments employing BS cooperation architectures and universal frequency reuse. We focus on the use of SC-FDE modulation schemes and IB-DFE based receivers for the detection process. Moreover, we considered the implementation of clustered techniques to simplify the detection procedure and to reduce the side information that must be 
transmitted by the backhaul network. The proposed methods depend on the interference level and from our results it can be concluded that, in the uplink transmission, it is possible to accurately detect the MTs with low detection requirements. When enabling an inter-cluster communication methodology, one can allow the flexibility of increasing the interference value and maintain a low overall system's detection requirement without sacrificing the performance.

\section{Acknowledgment}

This work was partially supported by the FCT (Fundação para a Ciência e a Tecnologia) under project UID/EEA/50008/2013 and Ph.D. grant SFRH/BD/87524/2012.

\section{References}

[1] C.-X. Wang, F. Haider, X. Gao, X.-H. You, Y. Yang, D. Yuan, H. Aggoune, H. Haas, S. Fletcher, E. Hepsaydir, Cellular architecture and key technologies for 5G wireless communication networks, IEEE Commun. Mag. 52 (2) (2014) $122-130$.

[2] E. Larsson, O. Edfors, F. Tufvesson, T. Marzetta, Massive MIMO for next generation wireless systems, IEEE Commun. Mag. 52 (2) (2014) 186-195.

[3] K. Balachandran, J.H. Kang, K. Karakayali, K.M. Rege, NICE: A network interference cancellation engine for opportunistic uplink cooperation in wireless networks, IEEE Trans. Wireless Commun. 10 (2) (2011) 540-549.

[4] K. Maruta, A. Ohta, M. Iizuka, T. Sugiyama, Iterative inter-cluster interference cancellation for cooperative base station systems, in: Vehicular Technology Conference, VTC Spring, 2012 IEEE 75th, May 2012, pp. 1-5.

[5] K. Balachandran, J.H. Kang, M.K. Karakayali, K.M. Rege, Base station cooperation with non-ideal backhaul and non-full buffer traffic, in: 2014 IEEE 80th Vehicular Technology Conference, VTC2014-Fall, Sept 2014, pp. 1-5.

[6] J. Wu, Z. Zhang, Y. Hong, Y. Wen, Cloud radio access network (C-RAN): A primer, IEEE Netw. 29 (1) (2015) 35-41.

[7] E. Bjo Andrnson, R. Zakhour, D. Gesbert, B. Ottersten, Cooperative multicell precoding: Rate region characterization and distributed strategies with instantaneous and statistical CSI, IEEE Trans. Signal Process. 58 (8) (2010) 4298-4310.

[8] D. Gesbert, S. Hanly, H. Huang, S. Shamai Shitz, O. Simeone, W. Yu, Multi-cell MIMO cooperative networks: A new look at interference, IEEE J. Sel. Areas Commun. 28 (9) (2010) 1380-1408.

[9] M. Sawahashi, Y. Kishiyama, A. Morimoto, D. Nishikawa, M. Tanno, Coordinated multipoint transmission/reception techniques for LTE-advanced [Coordinated and Distributed MIMO], IEEE Wirel. Commun. 17 (3) (2010) 26-34.

[10] O. Somekh, B. Zaidel, S. Shamai, Sum rate characterization of joint multiple cell-site processing, IEEE Trans. Inform. Theory 53 (12)(2007) 4473-4497.

[11] P. Silva, R. Dinis, Frequency-Domain Multiuser Detection for CDMA Systems, ser. River Publishers series in communications. Rajeev Ranjan Prasad, 2012. [Online]. Available: https://books.google.pt/books?id=s_5oqvS1hEQC.

[12] H. Sari, G. Karam, I. Jeanclaude, An analysis of orthogonal frequencydivision multiplexing for mobile radio applications, in: Vehicular Technology Conference, 1994 IEEE 44th, Vol. 3, Jun 1994, pp. 1635-1639.

[13] A. Gusmão, R. Dinis, J. Conceicão, N. Esteves, Comparison of two modulation choices for broadband wireless communications, in: Vehicular Technology Conference Proceedings, 2000. VTC 2000-Spring Tokyo. 2000 IEEE 51st, Vol. 2, May 2000, pp. 1300-1305.

[14] D. Falconer, S. Ariyavisitakul, A. Benyamin-Seeyar, B. Eidson, Frequency domain equalization for single-carrier broadband wireless systems, IEEE Commun. Mag. 40 (4) (2002) 58-66.

[15] R. Dinis, R. Kalbasi, D. Falconer, A. Banihashemi, Iterative layered space-time receivers for single-carrier transmission over severe time-dispersive channels, IEEE Commun. Lett. 8 (9) (2004) 579-581.

[16] N. Benvenuto, R. Dinis, D. Falconer, S. Tomasin, Single carrier modulation with nonlinear frequency domain equalization: An idea whose time has come again, Proc. IEEE 98 (1) (2010) 69-96.

[17] F. Silva, R. Dinis, N. Souto, P. Montezuma, Approaching the matched filter bound with block transmission techniques, Trans. Emerg. Telecommun. Technol. 23 (1) (2012) 76-85.
[18] M. Tüchler, J. Hagenauer, Linear time and frequency domain turbo equalization, in: IEEE Vehicular Technology Conference, 2001. VTC 2001 Fall., Vol. 4 2001, pp. 2773-2777.

[19] M. Tüchler, R. Koetter, A. Singer, Turbo equalization: principles and new results, IEEE Trans. Commun. 50 (5) (2002) 754-767.

[20] F.C. Ribeiro, R. Dinis, F. Cercas, A. Silva, Receiver design for the uplink of base station cooperation systems employing SC-FDE modulations, EURASIPJ. Wirel. Comm. Netw. 2015 (1) (2015)

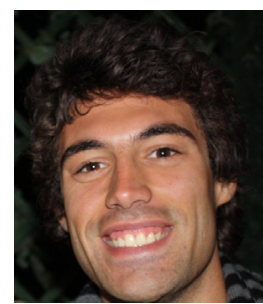

Filipe Casal Ribeiro received the M.S. degree in Telecommunications and Computer Science engineering from ISCTE - Lisbon University Institute, Lisbon, Portugal in 2012. He is currently working towards the Ph.D. degree in Information Science and Technology in ISCTE - Lisbon University Institute, Lisbon, Portugal and in the Wireless Communications group of Telecommunications Institute, Lisbon, Portugal, since 2013. His research interests include wireless communications systems and frequency-domain equalization issues in MIMO environments.

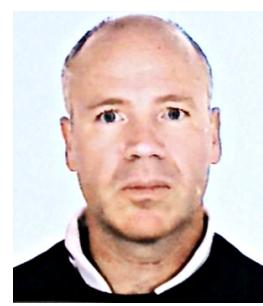

Rui Dinis received the Ph.D. degree from Instituto Superior Técnico (IST), Technical University of Lisbon, Portugal, in 2001. From 2001 to 2008 he was a Professor at IST. Since 2008 he is teaching at FCT-UNL (Faculdade de Ciências e Tecnologia da Universidade Nova de Lisboa). He was a researcher at CAPS/IST (Centro de Análise e Processamento de Sinais) from 1992 to 2005; from 2005 to 2008 he was researcher at ISR/IST (Instituto de Sistemas e Robótica); in 2009 he joined the research center IT (Instituto de Telecomunicações). He has been involved in several research projects in the broadband wireless communications area. His main research interests include modulation equalization, synchronization and channel estimation.

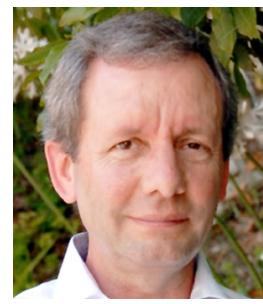

Francisco Cercas has more than 34 years of professional experience including more than 30 years of university teaching, 15 at IST and 16 at ISCTE-IUL where he is a Full Professor and the President of the Scientific Council. $\mathrm{He}$ is author and co-author of a new class of codes, TCH (Tomlinson, Cercas, Hughes), one patent, 4 book chapters, 14 journal papers, more than 150 conference papers and several research reports, $5 \mathrm{PhDs}$ theses supervision completed, as well as many MsC's and Final Year's Projects at both IST and ISCTE-IUL. He is Senior Member of IEEE, Coordinator of the Specialization in Telecommunications of Ordem dos Engenheiros since 2014 and Vice-coordinator of that Engineering Society between 2008 and 2013. He has been a researcher at CAPS, INESC, Satellite Centre of the University of Plymouth (UK) and Instituto de Telecomunicacões, where he is presently a Director of the IT Lisbon pole). His areas of interest include satellite communications, location and positioning, modulation and coding theory, mobile communications and related topics.

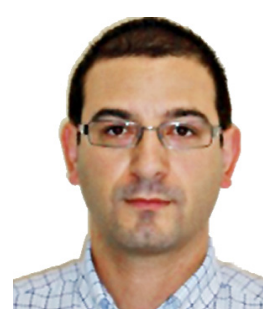

Adão Silva received the M.Sc. and Ph.D. degrees in electronics and telecommunications from the University of Aveiro in 2002 and 2007, respectively. He is currently an Assistant Professor in the Department of Electronics, Telecommunications and Informatics of the University of Aveiro, and a researcher at the Instituto de Telecomunicações. He has participated in several national and European projects, namely ASILUM, MATRICE, and 4MORE within the ICT programme, and the FUTON and CODIV projects with the FP7 ICT. He has led several research projects in the broadband wireless communications area at the national level. He has been a member of the TPC of several international conferences. His research interests include multiuser MIMO, multicarrier based systems, cooperative networks, precoding, and multiuser detection. 